\title{
Distributed Weighting Fusion and Covariance Intersection Fusion Kalman Smoother for Systems with Colored Measurement Noises
}

\author{
Peng Zhang \\ Department of Communication and Electronic Engineering \\ Qiqihar University \\ Qiqihar P.R. China \\ zhangpengxax@163.com
}

\author{
Wenjun Qi \\ School of Mechanical and Electrical Engineering \\ Heilongjiang University \\ Harbin P.R. China \\ 2015072@hlju.edu.cn
}

\begin{abstract}
For two-sensor system with colored measurement noises, based on the classical Kalman filtering method, distributed weighting fusion method and the covariance intersection (CI) fusion method, a CI fusion steady-state Kalman smoother is presented, which is independent of the unknown cross-covariance. It is difficult or complex to compute the crosscovariance in most cases. The accuracy comparison of the CI fusion Kalman smoother with three classical distributed weighting fusion Kalman smoothers which are weighted respectively by matrices, diagonal matrices, and scalars is proven, i.e., its accuracy is higher than that of each local Kalman smoother, and lower than that of optimal fuser weighted by matrices with known cross-covariance. The formula of the actual smoothing error variance of the CI fuser is given and the geometric interpretation of the above accuracy relations is presented based on the covariance ellipses. The Monte-Carlo simulation results show its effectiveness and correctness.
\end{abstract}

Keywords-Covariance intersection fusion; Kalman smoother; Cross-covariance; Accuracy relation; Covariance ellipse

\section{INTRODUCTION}

With the high-accuracy requirement of the estimation for the state or signal in many high-technology fields including tracking, defense, signal processing [1] and so on, the multisensor information fusion has been applied to these corresponding fields in recent years.

Unified fusion rules for the optimal linear estimation fusion and several distributed weighting state fusers were presented in [2-5], where the three distributed weighting fusers have the accuracy relations: the accuracy of the fuser weighted by matrices is higher than that of the fuser weighted by scalars, and the accuracy of the fuser weighted by diagonal matrices is between of them. However, all of the above weighting fusers have the limitation that in order to compute the optimal weights, the computation of the cross-covariances between the local estimation errors is required, while the cross-covariances are usually unknown [6] or their computation is very complex [7] in many applications. In order to overcome this limitation, the covariance intersection fusion method has been presented and developed in $[6,8,9]$ which can solve the fused filtering problems with unknown cross-covariances, and have the consistency and robustness. In this paper, a CI fused Kalman smoother is presented for the two-sensor system with colored measurement noises and with unknown cross-covariances, its accuracy relations among the local Kalman smoother, the CI fuser and three distributed weighting fusers are proven.

\section{THE LOCAL AND DISTRIBUTED WEIGHTING FUSION STEADY- STATE KALMAN SMOOTHERS}

Consider the two-sensor system with colored measurement noises

$$
\begin{gathered}
x(t+1)=\Phi x(t)+\Gamma w(t) \\
z_{i}(t)=H_{0} x(t)+\eta_{i}(t), i=1,2 \\
\eta_{i}(t+1)=c_{i} \eta_{i}(t)+\xi_{i}(t), i=1,2
\end{gathered}
$$

where $t$ is the discrete time, $x(t) \in R^{n}$ is the state, $Z_{i}(t) \in R^{m}$ is the ith measurement, $w(t) \in R^{m}, \eta_{i}(t) \in R^{m}$ are the input white noise and colored measurement noise, and $w(t)$ and $\xi_{i}(t)$ are independent white noise with zero mean and variance $Q_{w}$ and $Q_{\xi i}$, respectively. $\Phi, \Gamma, H_{0}, c_{i}$ are the matrices with corresponding dimensions.

The system (1), (2) and (3) can be transformed to the system with white noise by the corresponding transformation

$$
\begin{gathered}
x(t+1)=\Phi x(t)+\Gamma w(t) \\
y_{i}(t)=H_{i} x(t)+v_{i}(t), i=1,2
\end{gathered}
$$

where

$$
\begin{gathered}
y_{i}(t)=z_{i}(t+1)-c_{i} z_{i}(t), H_{i}=H_{0} \Phi-c_{i} H_{0}, \\
v_{i}(t)=H_{0} \Gamma w(t)+\xi_{i}(t), i=1,2
\end{gathered}
$$

Obviously, $v_{i}(t)$ is the white noise which is related to input noise $w(t)$, and $v_{i}(t)$ and $v_{j}(t)$ are also related when $i \neq j$.

$$
\begin{gathered}
\text { Define } \quad \mathrm{E}\left[v_{i}(t) v_{i}^{\mathrm{T}}(t)\right]=Q_{v i}, \quad \mathrm{E}\left[w(t) v_{i}^{\mathrm{T}}(t)\right]=S_{i} \\
\mathrm{E}\left[v_{i}(t) v_{j}^{\mathrm{T}}(t)\right]=R_{i j} \text {, we have } \\
\qquad \begin{array}{c}
Q_{v i}=Q_{w} H_{0} \Gamma \Gamma^{\mathrm{T}} H_{0}^{\mathrm{T}}+Q_{\xi i}, S_{i}=Q_{w} \Gamma^{\mathrm{T}} H_{0}^{\mathrm{T}}, \\
R_{i j}=Q_{w} H_{0} \Gamma \Gamma^{\mathrm{T}} H_{0}^{\mathrm{T}}, i \neq j
\end{array}
\end{gathered}
$$


The objectives are to find the local and CI fused steadystate Kalman smoothers $\hat{x}_{i}(t \mid t+N)$ and $\hat{x}_{C I}(t \mid t+N), N>0$ and the three distributed weighting fused steady-state Kalman smoothers $\hat{x}_{m}(t \mid t+N), \hat{x}_{d}(t \mid t+N), \hat{x}_{s}(t \mid t+N), N>0$, which means the weighted fuser weighted by matrices, diagonal matrices and scalars, respectively.

Lemma 1[10]. For the two-sensor system (4) and (5), the local steady-state Kalman state predictor $\hat{x}_{i}(t \mid t-1)$ of the ith sensor is given by

$$
\begin{gathered}
\hat{x}_{i}(t \mid t-1)=\Psi_{p i} \hat{x}_{i}(t-1 \mid t-2)+K_{p i} y_{i}(t-1) \\
\Psi_{p i}=\Phi-K_{p i} H, K_{p i}=\left(\Phi \Sigma_{i} H^{\mathrm{T}}+\Gamma S_{i}\right)\left(H \Sigma_{i} H^{\mathrm{T}}+Q_{v i}\right)^{-1}
\end{gathered}
$$

where the predicting error variance $\Sigma_{i}$ satisfies the steady-state Riccati equation

$$
\begin{gathered}
\Sigma_{i}=\Phi \Sigma_{i} \Phi^{\mathrm{T}}-\left[\Phi \Sigma_{i} H_{i}^{\mathrm{T}}+\Gamma S_{i}\right]\left[H_{i} \Sigma_{i} H_{i}^{\mathrm{T}}+Q_{v i}\right]^{-1} \times \\
{\left[\Phi \Sigma_{i} H_{i}^{\mathrm{T}}+\Gamma S_{i}\right]^{\mathrm{T}}+\Gamma Q_{w} \Gamma^{\mathrm{T}}}
\end{gathered}
$$

The steady-state predicting error crosscovariance $\Sigma_{i j}$ satisfies the following Lyapunov equation

$$
\Sigma_{i j}=\Psi_{p i} \Sigma_{i j} \Psi_{p j}^{\mathrm{T}}+\Delta_{p i j}, i \neq j, i, j=1,2
$$

where $\Delta_{p i j}=\Gamma Q_{w} \Gamma^{\mathrm{T}}-K_{p i} S_{i}^{\mathrm{T}} \Gamma^{\mathrm{T}}-\Gamma S_{j} K_{p j}^{\mathrm{T}}+K_{p i} R_{i j} K_{p j}^{\mathrm{T}}$.

Lemma 2[10]. For the two-sensor system (4) and (5), the local steady-state Kalman state smoother $\hat{x}_{i}(t \mid t+N), N>0$ of the $i$ th sensor is given by

$$
\hat{x}_{i}(t \mid t+N)=\hat{x}_{i}(t \mid t-1)+\sum_{k=0}^{N} K_{i}(k) \varepsilon_{i}(t+k), N>0
$$

where $\hat{x}_{i}(t \mid t-1)$ is obtained from (8), and

$$
\begin{gathered}
\varepsilon_{i}(t)=y_{i}(t)-H_{i} \hat{x}_{i}(t \mid t-1) \\
K_{i}(k)=\Sigma_{i} \Psi_{p i}^{\mathrm{Tk}} H^{\mathrm{T}}\left(H \Sigma_{i} H^{\mathrm{T}}+Q_{v i}\right)^{-1}, k=0, \cdots, N
\end{gathered}
$$

The steady-state smoothing error variance $P_{i}$ and crosscovariance $P_{i j}$ are given by

$$
\begin{aligned}
P_{i}= & \Sigma_{i}-\sum_{k=0}^{N} K_{i}(k)\left(H \Sigma_{i} H^{\mathrm{T}}+Q_{v i}\right)^{-1} K_{i}^{\mathrm{T}}(k), N>0 \\
P_{i j}= & \Sigma_{i j}-\sum_{r=0}^{N} K_{i}(r) H \Psi_{p i}^{r} \Sigma_{i j}-\sum_{s=0}^{N} \Sigma_{i j} \Psi_{p j}^{\mathrm{Ts}} H^{\mathrm{T}} K_{j}^{\mathrm{T}}(s)+ \\
& \sum_{r=0}^{N} \sum_{s=0}^{N} K_{i}(r) E_{i j}(r, s) K_{j}^{\mathrm{T}}(s), N>0
\end{aligned}
$$

where $\Sigma_{i}$ and $\Sigma_{i j}$ is obtained by (10) and (11). $E_{i j}(r, s)=\mathrm{E}\left[\varepsilon_{i}(t+r) \varepsilon_{j}^{\mathrm{T}}(t+s)\right]$ is as following

case 1 when $\min (r, s)>0$,

$$
\begin{aligned}
& \mathrm{E}_{i j}(r, s)=H_{i} \Psi_{p i}^{r} \Sigma_{i j} \Psi_{p j}^{\mathrm{Ts}} H_{j}^{\mathrm{T}}+ \\
& \sum_{k=1}^{\min (r, s)} H_{i} \Psi_{p i}^{r-k}\left[\begin{array}{ll}
\Gamma & -K_{p i}
\end{array}\right]\left[\begin{array}{ll}
Q_{w} & S_{j} \\
S_{i}^{\mathrm{T}} & R_{i j}
\end{array}\right]\left[\begin{array}{c}
\Gamma^{\mathrm{T}} \\
-K_{p j}^{\mathrm{T}}
\end{array}\right] \Psi_{p j}^{\mathrm{T}(s-k)} H_{j}^{\mathrm{T}}+R_{i j} \delta_{r s}
\end{aligned}
$$

case 2 when $\min (r, s)=0$,

$$
\mathrm{E}_{i j}(0,0)=H_{i} \Sigma_{i j} H_{j}^{\mathrm{T}}+R_{i j}
$$

$$
\begin{gathered}
\mathrm{E}_{i j}(r, 0)=H_{i} \Psi_{p i}^{r} \Sigma_{i j} H_{j}^{\mathrm{T}}+H_{i} \Psi_{p i}^{r-1}\left[\Gamma S_{j}-K_{p i} R_{i j}\right] \\
\mathrm{E}_{i j}(0, s)=H_{i} \Sigma_{i j} \Psi_{p j}^{\mathrm{Ts}} H_{j}^{\mathrm{T}}+\left[S_{i}^{\mathrm{T}} \Gamma^{\mathrm{T}}-R_{i j} K_{p j}^{\mathrm{T}}\right] \Psi_{p j}^{\mathrm{T}(s-1)} H_{j}^{\mathrm{T}}
\end{gathered}
$$

Lemma 3[6,10]. For the two-sensor system (4) and (5), the optimal fused Kalman smoother $\hat{x}_{m}(t \mid t+N), N>0$ weighted by matrices is given by

$$
\hat{x}_{m}(t \mid t+N)=\sum_{i=1}^{2} \Omega_{i} \hat{x}_{i}(t \mid t+N), N>0
$$

where the optimal weighted matrices $\Omega_{i}$ and fused error variance $P_{m}$ are given by

$$
\begin{gathered}
{\left[\Omega_{1}, \Omega_{2}\right]=\left(e_{m}^{\mathrm{T}} P^{-1} e_{m}\right)^{-1} e_{m}^{\mathrm{T}} P^{-1}, N>0} \\
P_{m}=\left(e_{m}^{\mathrm{T}} P^{-1} e_{m}\right)^{-1}, e_{m}^{\mathrm{T}}=\left[I_{m}, I_{m}\right], P=\left[\begin{array}{cc}
P_{1} & P_{12} \\
P_{12}^{\mathrm{T}} & P_{2}
\end{array}\right]
\end{gathered}
$$

The optimal Kalman smoother $\hat{x}_{s}(t \mid t+N), N>0$ weighted by scalars is given by

$$
\hat{x}_{s}(t \mid t+N)=\sum_{i=1}^{2} a_{i} \hat{x}_{i}(t \mid t+N), N>0
$$

where the optimal weighted coefficients $a=\left[\begin{array}{ll}a_{1} & a_{2}\end{array}\right]$ is given as

$$
a=\frac{e_{s}^{\mathrm{T}}\left(P_{\mathrm{tr}}\right)^{-1}}{e_{\mathrm{s}}^{\mathrm{T}}\left(P_{\mathrm{tr}}\right)^{-1} e_{s}}
$$

where

$$
\begin{gathered}
e_{\mathrm{s}}^{\mathrm{T}}=\left[\begin{array}{ll}
1 & 1
\end{array}\right] \\
P_{\mathrm{tr}}=\left[\begin{array}{cc}
\operatorname{tr} P_{1} & \operatorname{tr} P_{12} \\
\operatorname{tr} P_{21} & \operatorname{tr} P_{2}
\end{array}\right]
\end{gathered}
$$

The corresponding optimal fused error variance $P_{s}$ is given by

$$
P_{s}=\sum_{i=1}^{2} \sum_{j=1}^{2} a_{i} a_{j} P_{i j}
$$

The optimal Kalman smoother $\hat{x}_{d}(t \mid t+N), N>0$ weighted by diagonal matrices is given by

$$
\hat{x}_{d}(t \mid t+N)=\sum_{j=1}^{2} A_{i} \hat{x}_{i}(t \mid t+N)
$$

where the optimal weighted diagonal matrices $A_{j}$ is given as

$$
A_{j}=\left[\begin{array}{llll}
a_{j 1} & & & \\
& a_{j 2} & & \\
& & \ddots & \\
& & & a_{j n}
\end{array}\right], j=1,2
$$

The component expression of $\hat{x}_{j}$ and $\hat{x}_{d}$ are given by

$$
\hat{x}_{j}=\left[\begin{array}{c}
\hat{x}_{j 1} \\
\vdots \\
\hat{x}_{j n}
\end{array}\right], \hat{x}_{d}=\left[\begin{array}{c}
\hat{x}_{d 1} \\
\vdots \\
\hat{x}_{d n}
\end{array}\right]
$$

So the equation (29) is equivalent with 


$$
\hat{x}_{d l}(t \mid t+N)=\sum_{j=1}^{2} a_{j l} \hat{x}_{j l}(t \mid t+N), l=1, \cdots, n
$$

where the optimal weighted coefficient vector is

$$
a_{l}=\left[\begin{array}{ll}
a_{1 l} & a_{2 l}
\end{array}\right]=\frac{e_{d}^{\mathrm{T}}\left(P^{l l}\right)^{-1}}{e_{d}^{\mathrm{T}}\left(P^{l l}\right)^{-1} e_{d}}
$$

where $e_{d}^{\mathrm{T}}=\left[\begin{array}{lll}1 & \cdots & 1\end{array}\right]$ and defining

$$
P^{l l}=\left[\begin{array}{ll}
P_{1}^{(l l)} & P_{12}^{(l l)} \\
P_{21}^{(l l)} & P_{2}^{(l l)}
\end{array}\right]
$$

where $P_{i j}^{(l l)}$ is the $(l, l)$ th diagonal component of $P_{i j}$, and the optimal component fused error variance $P_{d l}$ and the optimal fused error variance $P_{d}$ are given by

$$
P_{d l}=\left[e_{d}^{\mathrm{T}}\left(P^{l l}\right)^{-1} e_{d}\right]^{-1}, l=1, \cdots, n, P_{d}=\sum_{i=1}^{2} \sum_{j=1}^{2} A_{i} P_{i j} A_{j}^{\mathrm{T}}
$$

\section{THE CI FUSED KALMAN SIGNAL SMOOTHER}

For the two-sensor system (4) and (5), when local steadystate filtering error variance $P_{i}$ is known, but the crosscovariance $P_{i j}$ is unknown, the CI fused Kalman smoother $\hat{x}_{C I}(t \mid t+N), N>0$ is given by

$$
\begin{aligned}
\hat{x}_{C I}(t \mid t+N)= & P_{C I}\left[\omega P_{1}^{-1} \hat{x}_{1}(t \mid t+N)\right. \\
& \left.+(1-\omega) P_{2}^{-1} \hat{x}_{2}(t \mid t+N)\right], N>0
\end{aligned}
$$

where $P_{C I}$ is defined as

$$
P_{C I}=\left[\omega P_{1}^{-1}+(1-\omega) P_{2}^{-1}\right]^{-1}
$$

where $\omega \in[0,1]$ and minimizes the performance index

$$
J=\min _{\omega} \operatorname{tr} P_{C I}=\min _{\omega \in[0,1]} \operatorname{tr}\left\{\left[\omega P_{1}^{-1}+(1-\omega) P_{2}^{-1}\right]^{-1}\right\}
$$

where the notation $\operatorname{tr}$ denotes the trace of matrix. The optimal weighted coefficient $\omega$ can be obtained by the gold section method or the Fabonacci method [11].

Defining $\bar{P}_{C I}$ is the actual smoothing error variance matrix of CI fused Kalman smoother, when $P_{i}$ is known, but $P_{i j}$ is unknown, the references $[6,9]$ have proven that $P_{C I}$ which is defined in (37) and (38) is an upper common bound of $\bar{P}_{C I}$, i.e.

$$
\bar{P}_{C I} \leq P_{C I}
$$

From (37), we can obtain $P_{C I}\left[\omega P_{1}^{-1}+(1-\omega) P_{2}^{-1}\right]=I$, which yields

$$
x(t)=P_{C I}\left[\omega P_{1}^{-1} x(t)+(1-\omega) P_{2}^{-1} x(t)\right]
$$

Subtracting (36) from (40), we can get the actual smoothing error

$$
\begin{aligned}
\tilde{x}_{C I}(t \mid t+N)= & P_{C I}\left[\omega P_{1}^{-1} \tilde{x}_{1}(t \mid t+N)\right. \\
& \left.+(1-\omega) P_{2}^{-1} \tilde{x}_{2}(t \mid t+N)\right], N>0
\end{aligned}
$$

The actual fused smoothing error variance $\bar{P}_{C I}=\mathrm{E}\left[\tilde{x}_{C I}(t \mid t+N) \tilde{x}_{C I}^{\mathrm{T}}(t \mid t+N)\right]$, where E denotes the mathematical expectation, $\mathrm{T}$ denotes the transpose. Substituting (41) into $\bar{P}_{C I}$ yields

$$
\begin{aligned}
\bar{P}_{C I}= & P_{C I}\left[\omega^{2}\left(P_{1}\right)^{-1}+\omega(1-\omega)\left(P_{1}\right)^{-1}\left(P_{12}\right)^{-1}\left(P_{2}\right)^{-1}\right. \\
& \left.\left.+\omega(1-\omega)\left(P_{2}\right)^{-1}\left(P_{21}\right)\right)^{-1}\left(P_{1}\right)^{-1}+(1-\omega)^{2}\left(P_{2}\right)^{-1}\right] P_{C I}
\end{aligned}
$$

\section{THE ACCURACY COMPARISON OF CI FUSED SMOOTHER AND} THREE WEIGHTED FUSED SMOOTHERS

Theorem 1. The local and fused Kalman smoothing error variance and cross-covariance have the relation as following

$$
P_{m} \leq \bar{P}_{C I}, \bar{P}_{C I} \leq P_{C I}, P_{m} \leq P_{i}, i=1,2, N>0
$$

Proof. According to the unbiasedness of local estimators $\hat{x}_{i}(t \mid t+N), i=1,2$, the fused estimator $\hat{x}_{m}(t \mid t+N)$ and $\hat{x}_{C I}(t \mid t+N), N>0$ are also unbiased. The linear minimum variance unbiased fused estimator weighted by matrices (22) has the property that its error variance matrix is less than or equal to any other linear unbiased estimation error variance matrix weighted by matrices, and (37) is a linear unbiased estimator weighted by matrices, so we have $P_{m} \leq P_{i}$ and $P_{m} \leq \bar{P}_{C I}$. References $[8,9,10]$ have proven that $\bar{P}_{C I} \leq P_{C I}$. The proof is completed.

Theorem 2. The local and fused Kalman smoothing error variances have the accuracy relation

$$
\begin{gathered}
\operatorname{tr} P_{m} \leq \operatorname{tr} P_{d} \leq \operatorname{tr} P_{s}, i=1,2, N>0 \\
\operatorname{tr} P_{m} \leq \operatorname{tr} \bar{P}_{C I} \leq \operatorname{tr} P_{C I} \leq \operatorname{tr} P_{i}, i=1,2, N>0
\end{gathered}
$$

Proof. reference [10] have proven that (44) holds. According to (39), taking $\omega=0$, we have $J=\operatorname{tr} P_{2}$, and taking $\omega=1$, we have $J=\operatorname{tr} P_{1}$. Hence the optimal weighting coefficient $\omega \in[0,1]$ yields $\operatorname{tr} P_{C I} \leq \operatorname{tr} P_{i}, i=1,2$. According to (43), $\operatorname{tr} P_{m} \leq \operatorname{tr} \bar{P}_{C I}, \operatorname{tr} \bar{P}_{C I} \leq \operatorname{tr} P_{C I}$, and (45) holds. The proof is completed.

\section{SiMULATION EXAMPLE}

Consider the two-sensor tracking system with colored measurement noises

$$
\begin{gathered}
x(t+1)=\Phi x(t)+\Gamma w(t) \\
z_{i}(t)=H_{0} x(t)+\eta_{i}(t), i=1,2 \\
\eta_{i}(t+1)=c_{i} \eta_{i}(t)+\xi_{i}(t), i=1,2
\end{gathered}
$$

where $w(t)$ and $\xi_{i}(t)$ are independent Gaussion white noises with zero mean and variances $Q_{w}$ and $Q_{\xi i}$, respectively. The problem is to obtain the local and fused smoother $\hat{x}_{i}(t \mid t+N), i=1,2, C I, m, s, d, N=1$, and compare their accuracy relations. In simulation, we take $T_{0}=0.5$, $\Phi=\left[\begin{array}{cc}1 & T_{0} \\ 0 & 1\end{array}\right], \Gamma=\left[\begin{array}{c}0.5 T_{0}^{2} \\ T_{0}\end{array}\right], H_{0}=\left[\begin{array}{ll}1 & 0\end{array}\right], c_{1}=0.85, c_{2}=0.1$, $Q_{w}=2.5, Q_{\xi 1}=0.16, Q_{\xi 2}=1.1$. Using gold section method, the CI fused weighting coefficient $\omega=0.41725$.

In order to give a geometric interpretation of the accuracy relations, the covariance ellipse for a variance matrix $P$ is 
defined as the locus of points $\left\{x: x^{T} P^{-1} x=c\right\}$ where $c$ is a constant. In the sequel, $c=1$ will be assumed without loss of generality. It was proved[6] that $P_{a} \leq P_{b}$ is equivalent to that the ellipse for $P_{a}$ is enclosed in the ellipse for $P_{b}$. The accuracy comparison by the covariance ellipses is shown in Fig 1 . From the accuracy relations (43), the ellipse for $P_{m}$ is enclosed in the ellipses for $\bar{P}_{C I}, P_{i}, i=1,2$, the ellipse for $\bar{P}_{C I}$ is enclosed in the ellipse for $P_{C I}$, and the ellipse for $P_{C I}$ encloses the intersection of the ellipses for $P_{1}$ and $P_{2}$ [9].

Define the mean square errors (MSE) at time $t$ as

$$
\begin{aligned}
\operatorname{MSE}_{i}(t) & =\frac{1}{M} \sum_{j=1}^{M}\left(\hat{x}_{i}^{(j)}(t \mid t+N)-x^{(j)}(t)\right)^{\mathrm{T}}\left(\hat{x}_{i}^{(j)}(t \mid t+N)-x^{(j)}(t)\right), \\
M & =300, i=1,2, C I, m, d, s
\end{aligned}
$$

where $\hat{x}_{i}^{(j)}(t \mid t+N)$ and $x^{(j)}(t)$ denote the $j$ th realization of $\hat{x}_{i}(t \mid t+N)$ and $x(t)$, respectively. According to the ergodicity of the sampled correlation function, it follows that $\operatorname{MSE}_{i}(t) \rightarrow \operatorname{tr} P_{i}, \operatorname{MSE}_{C I}(t) \rightarrow \operatorname{tr} P_{C I}$, as $M \rightarrow \infty, t \rightarrow \infty, i=1,2$

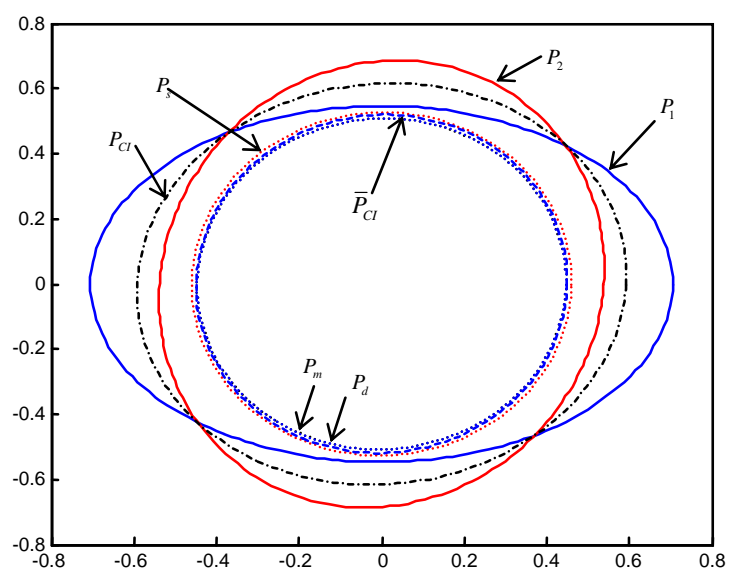

Fig. 1. The covariance ellipses for $P_{i}, i=1,2, m, d, s, C I$ and $\bar{P}_{C I}$

The results are shown in Fig 2., where the straight lines denote $\operatorname{tr} P_{i}, i=1,2, C I, m, d, s$, and $\operatorname{tr} \bar{P}_{C I}$, the curves denote the corresponding $\operatorname{MSE}_{i}(t)$. We see that the straight line $\operatorname{tr} P_{m}(0.45734)$ is very closed to the line $\operatorname{tr} P_{d}(0.45779)$, so the two straight lines are almost overlapping, and the accuracy relation (40) holds. Since the curve of $\operatorname{MSE}_{C I}(t)$ are close to the curve of $\operatorname{MSE}_{m}(t)$, so the actual accuracy of the CI fuser is close to that of the optimal fuser.

\section{CONCLUSION}

For the two-sensor system with colored measurement noises and with unknown cross-covariances, a CI fusion steady-state Kalman smoother is presented, which can avoid the computation of cross-covariances. It is proven that its accuracy is higher than that of each local smoother, and is a little lower than that of the optimal fuser weighted by matrices with known cross-covariances. The simulation results show that its accuracy is close to that of the optimal fuser weighted by matrices and diagonal matrices, so it has good performance.

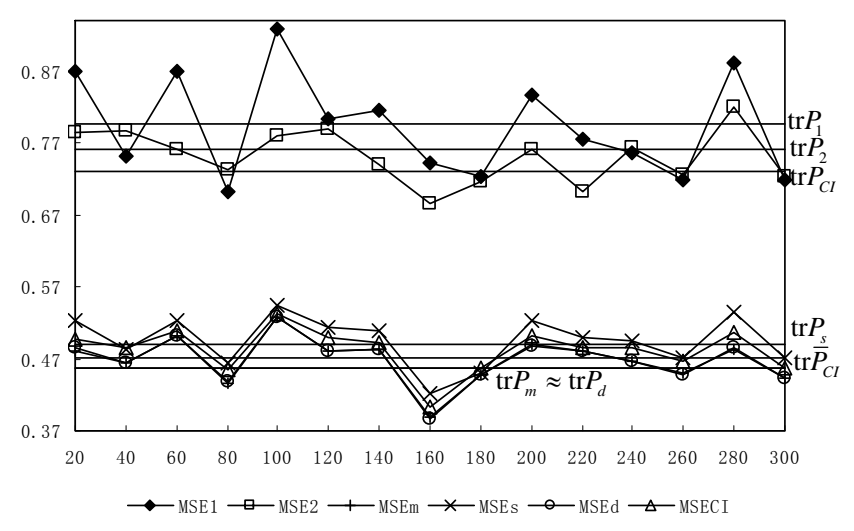

Fig. 2. The comparison of $\operatorname{MSE}_{i}(t)$ and $\operatorname{tr} P_{i}(i=1,2, C I, m, s, d)$ and $\operatorname{tr} \bar{P}_{C I}$

\section{Acknowledgment}

This work is supported by the Program for Young Teachers Scientific Research in Qiqihar University, 2014kM28(060356).

\section{References}

[1] Y. Bar-Shalom, X. R. Li, Estimation with applications to tracking and navigation. Thiagalingam Kirubarajan, John Wiley \& Sons. Inc, 2001.

[2] X. R. Li, Y. M. Zhu, J. Wang, C. J. Han, "Unified optimal linear estimation fusion, Part I: Unified fusion rule,” IEEE Trans. Information Theory, vol. 49(9), pp. 2192-2208, 2003.

[3] S. L. Sun, Z. L. Deng, "Multi-sensor optimal information Kalman filter," Automatica, vol. 40, pp. 1017-1023, 2004.

[4] Z. L. Deng, Y. Gao, L. Mao, Y. Li, G. Hao, "New approach to information fusion steady-state Kalman filtering," Automatica, vol. 41(10), pp. 1695-1707,2005.

[5] S. L. Sun, "Multi-sensor optimal information fusion Kalman filters with applications," Aerospace Science and Technology, vol. 8, pp. 57-62, 2004.

[6] S. J. Julier, J, K. Uhlman, "Non-divergent estimation algorithm in the presence of unknown correlations,” Proceedings of the IEEE American Control Conference, Albuquerque, NM, USA, pp. 2369-2373, 1997.

[7] X. J. Sun, Y. Gao, Z. L. Deng, C. Li, J. W. Wang, "Multi-model information fusion Kalman filtering and white noise deconvolution," Information Fusion, vol. 11, pp. 163-173, 2009.

[8] M. E. Liggins, D. L. Hall, J. Llinas, Handbook of Multisensor Data Fusion Theory and Practice, Second Edition.CRC Press, 2009.

[9] L. J. Chen, P. O. Arambel, C. Rago, R. K. Mehra, "Estimation under inknown correlation: Covariance intersection revisited," IEEE Trans. Automatic Control, vol. 47, pp. 1879-1882, 2002.

[10] Z. L. Deng, Multisensor information fusion filtering theory with applications. Harbin institute of technology Press, Harbin, 2007.

[11] Y. X. Yuan, W.Y. Sun, Optimization Theory and Method. Science Press, Beijing, 2003. 